\title{
Fachgruppe Differentielle Psychologie, Persönlichkeitspsychologie und Diagnostische Psychologie
}

\section{Weiterentwicklungen psychologischer Prüfungsformate aus diagnostischer Sicht}

\author{
Oliver Wilhelm¹, André Kretzschmar², Christian Montag ${ }^{1}$ und John Rauthmann ${ }^{3}$ \\ ${ }^{1}$ Institut für Psychologie und Pädagogik, Universität UIm \\ ${ }^{2}$ Psychologisches Institut, Universität Zürich, Schweiz \\ ${ }^{3}$ Abteilung Psychologie, Universität Bielefeld
}

Im Leitartikel „Ein Plädoyer zur Qualitätssicherung schriftlicher Prüfungen im Psychologiestudium" wird der Kenntnisstand der psychometrischen Forschung zur Konstruktion von Studien- und Prüfungsleistungen im Rahmen psychologischer Studiengänge kenntnisreich und profund dargelegt. Aus psychometrischer Sicht könnte man versucht sein zu argumentieren, dass nur wenig über das Offensichtliche hinausgegangen wird. Die abgeleiteten Konsequenzen für die Gestaltung schriftlicher Prüfungen werden nüchtern, empirisch gestützt abgewogen und gut verständlich vorgetragen. Bei genauerer Betrachtung hat der Leitartikel jedoch einige ernstzunehmende Konsequenzen. Wir wollen in diesem Beitrag (a) kurz auf die augenscheinlichen und naheliegenden Folgerungen für schriftliche und sonstige Prüfungen eingehen, (b) denkbare Weiterentwicklungen von Prüfungsformaten thematisieren und (c) anschließend allgemeiner über Prüfungen im Hochschulkontext aus diagnostischer und differenzieller Perspektive sprechen.

Die Gestaltung von Prüfungen mit geschlossenen Fragen liefert recht eindeutige Empfehlungen, von denen die Praxis in den Psychologiestudiengängen allerdings vielerorts spürbar abweicht. Abweichende Praktiken sind auch mikropolitischen Regelungen an einzelnen Universitäten oder landesrechtlichen Konkretisierungen bzgl. der Bewertung schriftlicher Prüfungen zuzuschreiben. Wünschenswert wäre, wenn sich die einschlägigen Regelungen am sachlich gebotenen Vorgehen orientieren würden - und hierfür liefert der Leitartikel von Lindner et al. hervorragende Begründungen und Empfehlungen. Insgesamt beschränken sich die Empfehlungen von Lindner et al. auf den klassischen Klausurkontext mit einem Fokus auf geschlossene Aufgabenformate (insbesondere Multiple-Choice-Aufgaben). Dies ist, wie im Leitartikel nach- 
vollziehbar dargelegt, dem Umstand geschuldet, dass Multiple-Choice-Aufgaben effizient sind und bei gegebener Expertise mit überschaubarem Aufwand objektive, reliable und valide Prüfungen erlauben. In der Praxis gelten akademische Abschlussarbeiten, ausformulierte Seminararbeiten u. Ä. aber ebenfalls als schriftliche Prüfungsleistungen, die zur Diagnostik in Frage stehender akademischer Kompetenzen möglicherweise besser geeignet oder angemessener sind. So wie Lindner et al. Empfehlungen für schriftliche Prüfungen (insbesondere für geschlossene Aufgabenformate) aussprechen, wäre es wünschenswert, auch handlungsnahe Leitlinien für weitere schriftliche Prüfungsformen sowie mündliche Prüfungen zu erarbeiten. Ergänzend wäre es wünschenswert, die methodischen Besonderheiten bestimmter Prüfungsformate empirisch verstärkt zu untersuchen. Schließlich ist festzuhalten, dass praktische Prüfungen, einschließlich ihrer neueren Ausdeklinierungen (insbesondere objektiv strukturierte klinische Prüfungen), gerade im Kontext der Therapieausbildung erheblich an Bedeutung gewinnen werden. Methoden der Leistungsbewertung und Aspekte der Aufgabengestaltung sind hier ebenso forschungswürdig wie die Konkretisierung der inhaltlichen Beschaffenheit solcher Prüfungen.

Ein wesentlicher Aspekt, der zukünftig vermutlich höheres Gewicht erhält und durch die COVID-19 Pandemie beschleunigt werden könnte, sind digitalisierte Prüfungsformate. Wenig Gedanken muss man sich machen, wenn herkömmliche Prüfungen praktisch unverändert auf den Computer übertragen werden solange kein starker Zeitdruck bei der Bearbeitung vorliegt und Studierende in einer Gruppentestung beaufsichtigt werden können (z. B. in einem Computerraum). Schwieriger wird es dagegen, schriftliche Einzel- oder Gruppenprüfungen in digitaler Form in einem privaten Umfeld $\mathrm{zu}$ ermöglichen. Der Schutz von Prüfungsfragen vor ungewollter Exposition, die Verwendung unerlaubter Hilfsmittel und die Sicherstellung gleichermaßen zuträglicher Testbedingungen für alle Personen sind auf der Hand liegende Herausforderungen. Zwischen traditionell beaufsichtigten Präsenzprüfungen und digital realisierten selbstadministrierten Prüfungen sind zahllose Abstufungen denkbar, deren Brauchbarkeit für die Vergabe akademischer Zertifikate empirischer Überprüfung harrt.

Darüber hinaus ergeben sich durch neue digitale Prüfungsformen auch neue juristische Herausforderungen, etwa bei technischen Fehlfunktionen, die bei Papier-Stift Prüfungen kaum drohen. Aus diagnostischer Sicht sind im Hochschulsektor digitale Prüfungen jedoch deutlich unterentwickelt, und es liegt ein spürbares Defizit vor.

Methodische Perspektiven wie adaptives Testen und unmittelbare, auch aufgeschlüsselte Leistungsrückmeldung lassen sich ebenfalls als je nach Kontext verschie- den stark ins Gewicht fallende Neuerungen verstehen. Die Beschaffenheit schriftlicher Prüfungen könnte stärker dadurch verändert werden, dass Prüfungsdaten unmittelbarer zugänglich sind, automatisiert aufbereitet werden könnten und folglich eine stärkere Rückmeldefunktion für Unterricht und Prüfung entwickeln könnten. Bedeutsam scheint uns auch, dass neue Technologien neue Perspektiven auf Leistungsprüfungen erlauben. Dies mag Entwicklungen wie sogenannte stealth-Prüfungen, Leistungsmessungen in virtuellen Kontexten, gamifizierte Prüfungen, kollaborative schriftliche Leistungen, teilautomatisierte Bewertung des semantischen Inhalts offener Antworten u. Ä. enthalten. Erfreulich wäre, wenn psychologische Forschung hier mehr erreichen könnte als technologische Entwicklungen nur im Nachgang zu adaptieren. Gleichzeitig möchten wir darauf hinweisen, dass es primär nicht darum gehen sollte, „neue Darstellungsformen" und komplexere Aufgaben nur deshalb umzusetzen, weil es technisch möglich ist und man sich eine vermeintlich höhere Augenscheinvalidität oder ökologische Validität erhofft. Vielmehr ist aus einer kognitiven Anforderungsperspektive heraus zu begründen und empirisch aufzuzeigen, welche Kompetenzen zusätzlich oder besser durch neue Aufgabenformate abgebildet werden können. Ferner sollten dabei auch keine unbeabsichtigten Benachteiligungen (z. B. aufgrund von zusätzlichen kognitiven Anforderungen an die Informations- und Technologieliteratheit) eingeführt werden. Folglich ist hier empirische Forschung über verschiedene, insbesondere auch technologiebasierte Prüfungsformate in- und außerhalb der Psychologie nötig.

Es ist eine Illusion zu glauben, dass im realen hochschulischen Berufskontext die quantitative Betrachtung schriftlicher Prüfungsleistungen eine nennenswerte Rolle spielen würde. Eine Curriculumsorientierung in der Prüfungserstellung wird hingegen in aller Regel gegeben sein. Damit ist allerdings nicht gesagt, dass Prüfungen, die an verschiedenen Hochschulen die (im Wesentlichen) gleiche Bezeichnung tragen, auch inhaltlich gleichartig beschaffen sind. Neben der Frage, ob im Wesentlichen gleich Beschaffenes auch im Wesentlichen gleich geprüft wird, ist auch die Frage der Milde oder Strenge von Bewertungsmaßstäben im Großen und Ganzen unbekannt. Für die Kohärenz eines Faches wäre ein einheitliches Kerncurriculum eigentlich wichtig; allerdings ist dies praktisch gesehen nicht einfach herzustellen, da eine Profilbildung in der Lehre vielerorts gewünscht und an den Forschungsstärken von Fachbereichen orientiert sein wird.

So wie die Pädagogische Psychologie seit Jahrzehnten schulische Leistungsprüfungen erforscht und die Arbeitsund Organisationspsychologie routinemäßige Dienstleistungen, die auf Leistungsprüfungen beruhen, im Arbeits- 
kontext anbietet, sollte auch im Hochschulkontext die evaluative Begleitung von Prüfungen selbstverständlich sein. Bei der Anerkennung von Prüfungsleistungen für Studienortwechsler_innen, der Formulierung von Voraussetzungen in Zulassungsordnungen oder der Anerkennung der Gleichwertigkeit von Studienabschlüssen wird eine hinreichende Kohärenz der in Frage stehenden Leistungen (oft implizit) angenommen. Inwiefern die Situation bei zentral organisierten und hochschulextern überwachten Prüfungen wissenschaftlich belastbarer ist, kann nur als empirische Frage begriffen werden. Wir verstehen diese Frage als eine Herausforderung, die in der wissenschaftlichen Gemeinde mehr Beachtung verdient. Die praktischen Konsequenzen (etwa die Zertifizierung als Psychotherapeut_in) sind fachlich von so großer Bedeutung, dass eine routinemäßige Evaluation eines solchen Prüfungsgeschehens unumgänglich ist.

Aus diagnostischer Sicht spricht zunächst wenig gegen eine einheitliche Überprüfung der Kompetenzen in einem Kerncurriculum. Ein Kerncurriculum muss dabei nicht strikt und top-down alle möglichen Inhalte, Phänomene, Fragen, Effekte etc. vorgeben und sollte auch nicht als statischer Kanon aufgefasst werden. Es macht jedoch Sinn, unumgängliche „Kerne“ eines Faches zu identifizieren und korrespondierende Kompetenzen ergebnisorientiert zu prüfen. Beispielsweise kann ein Diagnostikcurriculum schwerlich auf die Behandlung gängiger Messmodelle verzichten. Die Erörterung der genauen Bestandteile eines Kerncurriculums kann unseres Erachtens ein wichtiger Baustein für die Stiftung von Kohärenz innerhalb eines Faches sein. Die beteiligten Fachleute sollten bei der Erarbeitung eines Vorschlages gewiss im Blick behalten, dass die individuellen Unterschiede in der Kriteriumserreichung grundsätzlich Daten für Standortevaluationen hinsichtlich des Lernerfolgs enthalten.

Aus diagnostischer Perspektive konstituiert eine Outputorientierung, wie sie im Gefolge des PISA-Schocks in anderen Bildungsbereichen bereits vor einiger Zeit erfolgte, auch im tertiären Bildungsbereich einen Fortschritt. Die Psychologie ist die Disziplin, die die Brauchbarkeit akademischer Prüfungen wissenschaftlich untersuchen kann, und sie sollte sich selbst nicht ausnehmen, wenn es um die Betrachtung entsprechender Daten geht.

\section{Prof. Dr. Oliver Wilhelm}

Institut für Psychologie

Universität Ulm

89069 Ulm

oliver.wilhelm@uni-ulm.de

https://doi.org/10.1026/0033-3042/a000526 